\title{
Astroparticle Physics at DUNE
}

\section{Inés Gil-Botella*†}

Centro de Investigaciones Energéticas, Medioambientales y Tecnológicas (CIEMAT)

E-mail: ines.gileciemat.es

\begin{abstract}
The Deep Underground Neutrino Experiment (DUNE) will pursue a broad program on neutrino physics and astrophysics with a staged $40 \mathrm{kt}$ LAr detector at the Sanford Underground Research Facility (SURF) $1300 \mathrm{~km}$ from Fermilab. The scientific goals include neutrino oscillations studies with a powerful neutrino beam from Fermilab leading to determining the neutrino mass ordering and the measurement of the $\mathrm{CP}$ violating phase. The underground location of the DUNE far detector will allow undertaking searches for new phenomena and the detection of astrophysical processes involving neutrino emission. The observation of the neutrino signal from a core-collapse supernova in the DUNE LAr TPCs will provide unique and unprecedented information on the mechanism of supernovae in addition to enabling the search for new physics. In addition, DUNE will search for proton decay in the range of proton lifetimes predicted by a wide range of GUT models by virtue of its high detection efficiency and low background rates.
\end{abstract}

The European Physical Society Conference on High Energy Physics

5-12 July, 2017

Venice

\footnotetext{
*Speaker.

$\dagger$ on behalf of the DUNE collaboration.
} 


\section{The Deep Underground Neutrino Experiment (DUNE)}

The DUNE/LBNF [1] program aims to address key questions in neutrino physics and astroparticle physics. It includes precision measurements of the parameters that govern neutrino oscillations with the goal of measuring the $\mathrm{CP}$ violating phase and the neutrino mass ordering, nucleon decay searches and detection and measurement of the electron neutrino flux from a core-collapse supernova within our galaxy. The ancillary program for underground physics in DUNE also includes the measurement of neutrino oscillations using atmospheric neutrinos, solar neutrinos, detecting diffuse supernova neutrino fluxes and searches for neutrinos from extra-solar astrophysical sources.

DUNE will consist of a near detector placed at Fermilab close to the neutrino beam and four 10 kt fiducial mass LAr TPCs as far detector at $1300 \mathrm{~km}$ from Fermilab in the Sanford Underground Research Facility (SURF) at 4300 mwe depth. The deep location of the DUNE far detector will enable non-beam physics measurements including supernova neutrinos and nucleon decay searches.

\section{Supernova Neutrino Detection in DUNE}

Core-collapse supernovae ( $\mathrm{SN}$ ) are a huge source of neutrinos of all flavors in our Universe. During a SN explosion, $99 \%$ of the gravitational binding energy of the star (of the order of $3 \times 10^{53}$ ergs) is released by neutrinos and antineutrinos of all flavors, which play the role of astrophysical messengers, escaping from the SN core.

Neutrinos from core-collapse supernovae are emitted in a burst of a few tens of seconds duration [2]. Essentially three stages can be distinguished: the neutronization burst, a large $v_{e}$ emission in the first 10's of ms, the accretion phase, which lasts between few tens to few hundreds of ms, where the luminosity of electron and non-electron flavors is significantly different, and the cooling phase, up to $\sim 10 \mathrm{~s}$, when luminosities and average energies decrease and there is almost luminosity equipartition between neutrino species. MSW flavor transitions and other neutrino collective effects may modify significantly the expected supernova neutrino spectra [3]. By measuring the flavor composition, energy spectrum and time structure of the neutrino burst, critical information about the SN dynamics and neutrino properties as neutrino mass ordering can be obtained.

Liquid Argon TPCs have excellent sensitivity to electron neutrinos from SN. This technology provides good energy resolution and full particle reconstruction with very high quality tracking. Energy thresholds as low as a few $\mathrm{MeV}$ may be possible. In these detectors, the ionization charge is drifted by an electric field towards the anode where the charge is collected. Using the time arrival of the charge at the readout planes, a three-dimensional track reconstruction is possible. Particles are identified by the rate of energy loss along the track. The Ar scintillation light is also detected enabling fast timing of signals and event localization inside the detector.

At low energy, neutrinos can be detected in LAr through four detection channels:

1. Charged-current (CC) interactions on $\operatorname{Ar}\left(v_{e}+{ }^{40} \mathrm{Ar} \rightarrow e^{-}+{ }^{40} \mathrm{~K}^{*}\right.$ and $\left.\bar{v}_{e}+{ }^{40} \mathrm{Ar} \rightarrow e^{+}+{ }^{40} \mathrm{Cl} l^{*}\right)$. The neutrino energy thresholds of these reactions are 1.5 and $7.48 \mathrm{MeV}$, respectively.

2. Neutral-current (NC) interactions on Ar for all flavor neutrinos $\left(\vec{v}+{ }^{40} A r \rightarrow\left(\vec{v}+{ }^{40} A r^{*}\right)\right.$. The energy threshold of this reaction is $1.46 \mathrm{MeV}$.

3. Elastic scattering (ES) on atomic electrons for all flavor neutrinos $\left(\vec{v}+e^{-} \rightarrow(\vec{v})+e^{-}\right)$. 
Fig. 1 shows the cross sections of all the processes as a function of the neutrino energy [4]. It is possible to separate the different channels by measuring the associated photons coming from the de-excitation of $\mathrm{K}, \mathrm{Cl}$ and $\mathrm{Ar}$ or by the absence of photons in the case of elastic scattering. It is important to have a good knowledge of the final state of the interactions, specially the de-excitation $\gamma_{\mathrm{S}}$ from the ${ }^{40} \mathrm{~K}$. However, precision models of low energy neutrino argon reactions or dedicated measurements are not available yet.

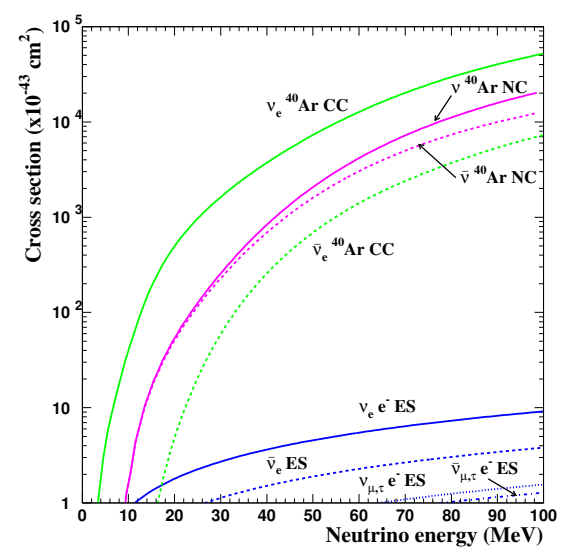

Figure 1: Neutrino cross sections relevant to the supernova detection with a liquid argon TPC [4].

One of the primary scientific goals of DUNE is the detection of the burst of neutrinos of all flavors from a core-collapse supernova, of which the component detectable by the DUNE LArTPC is primarily $v_{e}$. Table 1 shows the rates for the dominant interactions in argon for the Livermore model [5] (included for comparison with literature), and the GKVM model [6]; for the former, no oscillations are assumed; the latter assumes collective effects. In general, there is a rather wide variation, up to an order of magnitude, in event rate for different models.

\begin{tabular}{lcc} 
Channel & Events Liv. model & Events GKVM model \\
\hline$v_{e}+{ }^{40} \mathrm{Ar} \rightarrow e^{-}+{ }^{40} \mathrm{~K}^{*}$ & 2720 & 3350 \\
$\bar{v}_{e}+{ }^{40} \mathrm{Ar} \rightarrow e^{+}+{ }^{40} C l^{*}$ & 230 & 160 \\
$\left(\vec{v}+e^{-} \rightarrow\left(\vec{v}+e^{-}\right.\right.$ & 350 & 260 \\
\hline
\end{tabular}

Table 1: Event rates in DUNE for a core-collapse SN at $10 \mathrm{kpc}$.

Fig. 2 shows the time profile and the expected energy spectrum for the different CC and ES detection channels in DUNE for a SN at $10 \mathrm{kpc}$. No oscillations have been considered. It is evident the clear dominance of the $v_{e}$ channel and the huge visibility of the neutronization burst.

LAr TPCs can provide unique information about the early breakout pulse from the detection of $v_{e}$ neutrinos mainly through the CC process. The advantage of the early phase analysis is that is not affected by the time evolution of the density structure of the star or whether the remnant is a neutron star or a black hole. The analysis of the time structure of the supernova signal during the first few tens of milliseconds after the core bounce can provide a clean indication if the full $v_{e}$ burst is present or absent and therefore allows distinguishing between different mixing scenarios. Fig. 3 shows the effect of the reduction of the $v_{e}$ peak due to oscillations. The suppression is maximal 

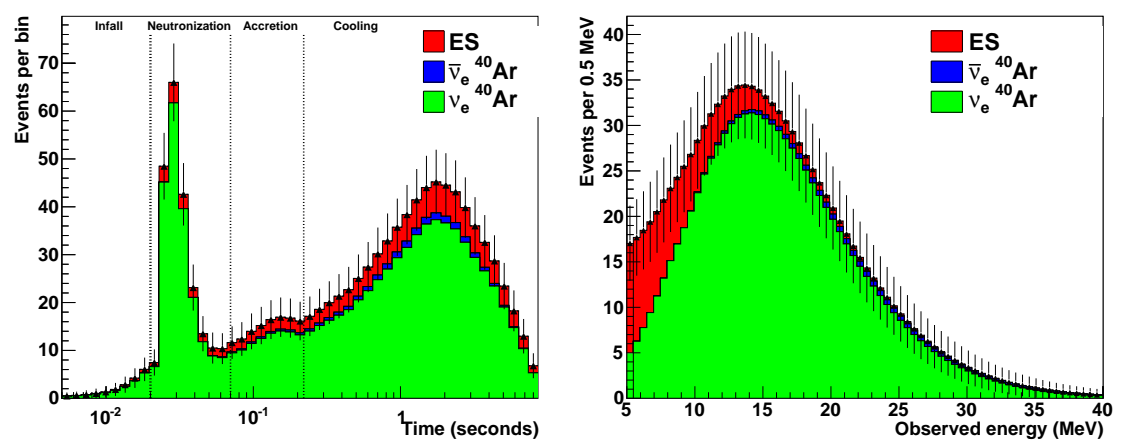

Figure 2: Left: Expected time-dependent signal in $40 \mathrm{kt}$ of liquid argon for an electron-capture supernova [7] at $10 \mathrm{kpc}$, calculated using SNOwGLoBES [8], showing breakdown of event channels. Right: Expected measured event spectrum for the same model, integrated over time.

for normal hierarchy due to the total conversion of $v_{e}$ into $v_{\mu, \tau}$. The total number of events per bin (dominated by electron neutrino flavor) is shown in logarithmic bins.

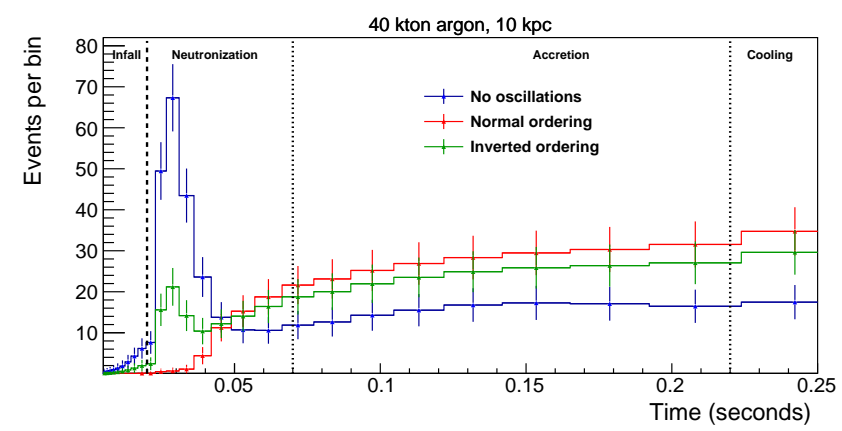

Figure 3: Time evolution of the neutrino signal for a supernova at $10 \mathrm{kpc}$ in $40 \mathrm{kt}$ of argon up to the start of the cooling phase for different MSW oscillation scenarios.

\section{Nucleon Decay Searches}

Grand Unified Theories (GUT) make predictions about baryon number violation and proton lifetime that may be within reach of DUNE. There is no evidence for proton decay in Nature and the lifetime limits from the current generation of experiments already constrain many GUT models. In some cases, the experimental limits are approaching the upper bounds of what these models will allow. The new generation of large underground experiments will try to improve the sensitivity to specific proton decay modes favored by GUT models. In particular, the imaging, particle identification and calorimetric capabilities of LAr TPCs will allow to improve the sensitivity to many decay channels. Multi-prong decay modes like e.g. $p \rightarrow \mu \pi^{+} K^{+}$or $p \rightarrow e^{+} \pi^{+} \pi^{-}$and channels involving kaons like e.g. $p \rightarrow K^{+} \bar{v}, p \rightarrow e^{+} K^{0}$ and $p \rightarrow \mu^{+} K^{0}$ are particularly suitable, since liquid Argon imaging provides typically an order of magnitude improvement in efficiencies for similar or better background conditions compared to Water Cerenkov detectors. 
Fig. 4 shows a reconstructed $p \rightarrow K^{+} \bar{v}$ event simulated in DUNE and identified by analysis selection criteria, where $K^{+} \rightarrow \mu^{+} v_{\mu}$ and $\mu^{+} \rightarrow e^{+} v_{e} \bar{v}_{\mu}$. This decay mode is uniquely interesting for DUNE since stopping kaons have a higher ionization density than lower-mass particles and a LArTPC could identify the $\mathrm{K}^{+}$track with high efficiency. Fig. 4 also shows how each of these particles would be identified using a PIDA algorithm [9].
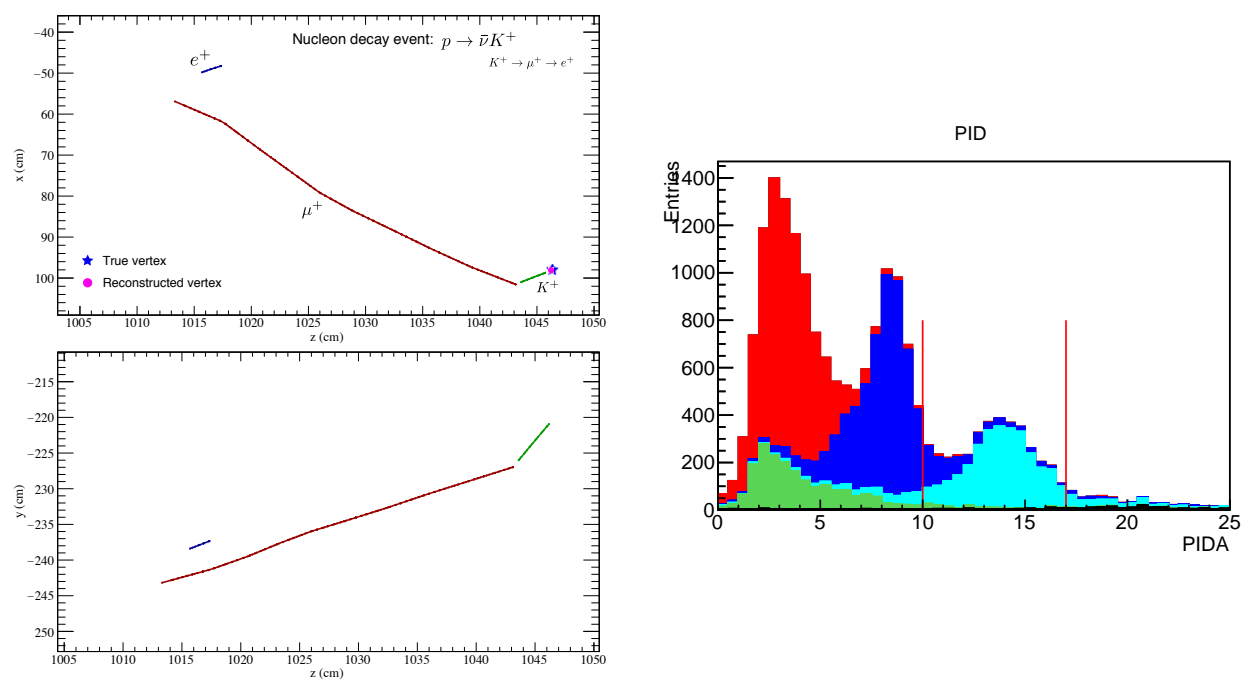

Figure 4: (Left) Fully reconstructed $p \rightarrow K^{+} \bar{v}$ event simulated in DUNE. The drift direction is parallel to the $x$ axis. (Right) PIDA distribution for all tracks in $p \rightarrow K^{+} \bar{v}$ sample. Different colors indicate different particle types: protons (black), charged kaons (cyan), muons (blue), electrons (red) and other particles (green). The vertical red lines indicate the cut values chosen to select kaons in the analysis.

Fig. 5 shows DUNE's expected partial lifetime sensitivity to $p \rightarrow K^{+} \bar{v}$ as a function of signal efficiency and background rate, for a $400 \mathrm{kt} \cdot$ year exposure and at $90 \%$ confidence level. Assuming a 54\% signal efficiency in background-free conditions, the partial lifetime sensitivity will be $2.4 \times$ $10^{34} \mathrm{yr}$ for a $400 \mathrm{kt} \cdot$ year exposure and at $90 \% \mathrm{CL}$. This sensitivity is 4 times better than the current Super-Kamiokande limit [10].

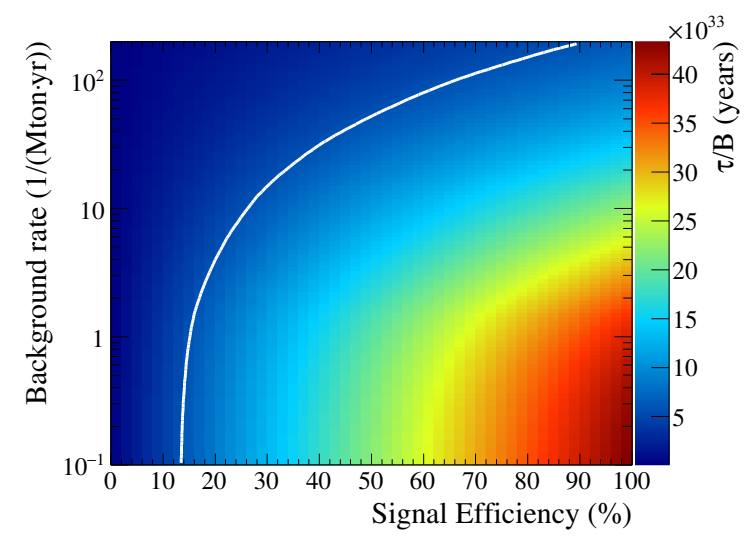

Figure 5: Partial lifetime sensitivity at DUNE for a $400 \mathrm{kt} \cdot$ year exposure at $90 \% \mathrm{CL}$, as a function of the background rate and signal efficiency. The white line shows corresponds to the Super-K current limit. 


\section{References}

[1] R. Acciarri et al., [DUNE Collaboration], arXiv:1512.06148

[2] A. Mirizzi et al., Nuovo Cim. 39, N. 1-2 (2016)

[3] H. Duan and F. Friedland, Phys. Rev. Lett. 106 (2011) 091101

[4] I. Gil-Botella and A. Rubbia, JCAP 0408 (2004) 001; JCAP 10 (2003) 009; A. Bueno, I. Gil-Botella and A. Rubbia, hep-ph/0307222

[5] T. Totani et al., Astrophys. J. 496 (1998) 216

[6] J. Gava et al., Phys. Rev. Lett. 103 (2009) 071101

[7] L. Hudepohl et al., Phys. Rev. Lett. 104 ()2010 251101

[8] http://www.phy.duke.edu/ schol/snowglobes/

[9] R. Acciarri et al., [ArgoNeuT Collaboration], JINST 8 (2013) P08005

[10] K. Abe et al., [Super-Kamiokande Collaboration], Phys. Rev. D 90 (2014) no.7, 072005 